\title{
Recolección de guano de murciélagos de Cabrera (Pipistrellus pygmaeus) por hormigas rojas (Formica rufa)
}

\author{
JuAn TOMÁs AlCALDE ${ }^{1 *}$, INMACUladA AnTóN
}

${ }^{1}$ Plaza Sabicas 5, 2º B. 31015, Pamplona, Spain.
${ }^{*}$ Corresponding author e-mail: jtalcalde@gmail.com

DOI: http://dx.doi.org/10.14709/BarbJ.7.1.2014.06

English title: Collecting bat guano of soprano pipistrelles (Pipistrellus pygmaeus) by red wood ants (Formica rufa)

\begin{abstract}
In June 1999, a colony of soprano pipistrelles (Pipistrellus pygmaeus Leach, 1825) was found in a dead white poplar (Populus alba L., 1753) in Lodosa, Navarre (Northern Spain). More than 717 bats roosted in the same tree. This tree was cut down, and the colony finally disappeared. In 2003, six bat-boxes (Schwegler, 1FF model) were placed in the same area by the regional authorities (Dept. of Environment, Gov. of Navarre) to provide alternative roosts for this species. In July 2008, five bat-boxes were occupied by new breeding colonies of soprano pipistrelles, ranging approximately from 20 to 40 individuals each one. This year, we observed that many red wood ants (Formica rufa L., 1761) collected the bat guano from the ground, below one bat-box, and they took it up the tree trunk, to their nest, located $2 \mathrm{~m}$ above the bat-box. Similar behavior has been mentioned by other researchers for some different ant species and other insects, but until now, always in caves. As red wood ants normally feed on other insects, we think that they could collect bat guano as a food resource, in an opportunistic behavior. Thus, soprano pipistrelles have a positive effect on the ant colony.
\end{abstract}

Keywords: Guano, Formica rufa, bat boxes, Pipistrellus pygmaeus.

En julio de 1999 se localizó una numerosa colonia reproductora de murciélagos de Cabrera, (Pipistrellus pygmaeus Leach, 1825) refugiada en un álamo (Populus alba L., 1753) viejo de Lodosa (Navarra, norte de España), a las orillas de río Ebro.

El árbol refugio fue talado en agosto de ese mismo año y la colonia, de más de 717 ejemplares, desapareció (Alcalde 2000). En 2003, el Departamento de Medio Ambiente del Gobierno de Navarra decidió la colocación de cajas-refugio en árboles de las cercanías, para compensar la pérdida del refugio original y favorecer la permanencia de la población de murciélagos de Cabrera en la zona. Se instalaron 6 cajas planas, del modelo 1FF de la empresa Schwegler. Al carecer de base, estos refugios dejan caer libremente el guano al suelo.

En julio de 2008, cinco cajas estaban ocupadas por pequeñas colonias reproductoras de entre 20 y 40 individuos adultos aproximadamente. Durante una revisión llevada a cabo el 6 de septiembre del mismo año, se comprobó que todas las cajas seguían estando habitadas por grupos de murciélagos. Bajo todas ellas, a excepción de una, se apreciaba una patente mancha de guano, procedente de las agrupaciones situadas unos metros más arriba. Una observación más detallada en la zona limpia que quedaba debajo de la caja mencionada reveló la presencia de numerosas hormigas rojas (Formica rufa L., 1761) inspeccionando el terreno. Se pudo comprobar también que algunas hormigas recogían el guano fresco, recién caído (Fig. 1), y lo transportaban por el tronco del árbol hasta una grieta situada a $7 \mathrm{~m}$ de altura, $2 \mathrm{~m}$ por encima de la caja donde se encontraban los murciélagos (Fig. 2). Como consecuencia, el suelo se encontraba casi totalmente vacío de guano a pesar de que la caja contenía en ese momento un mínimo de 13 murciélagos de Cabrera.

Algunos autores mencionan comportamientos similares, aunque siempre referidos a especies cavernícolas como por 


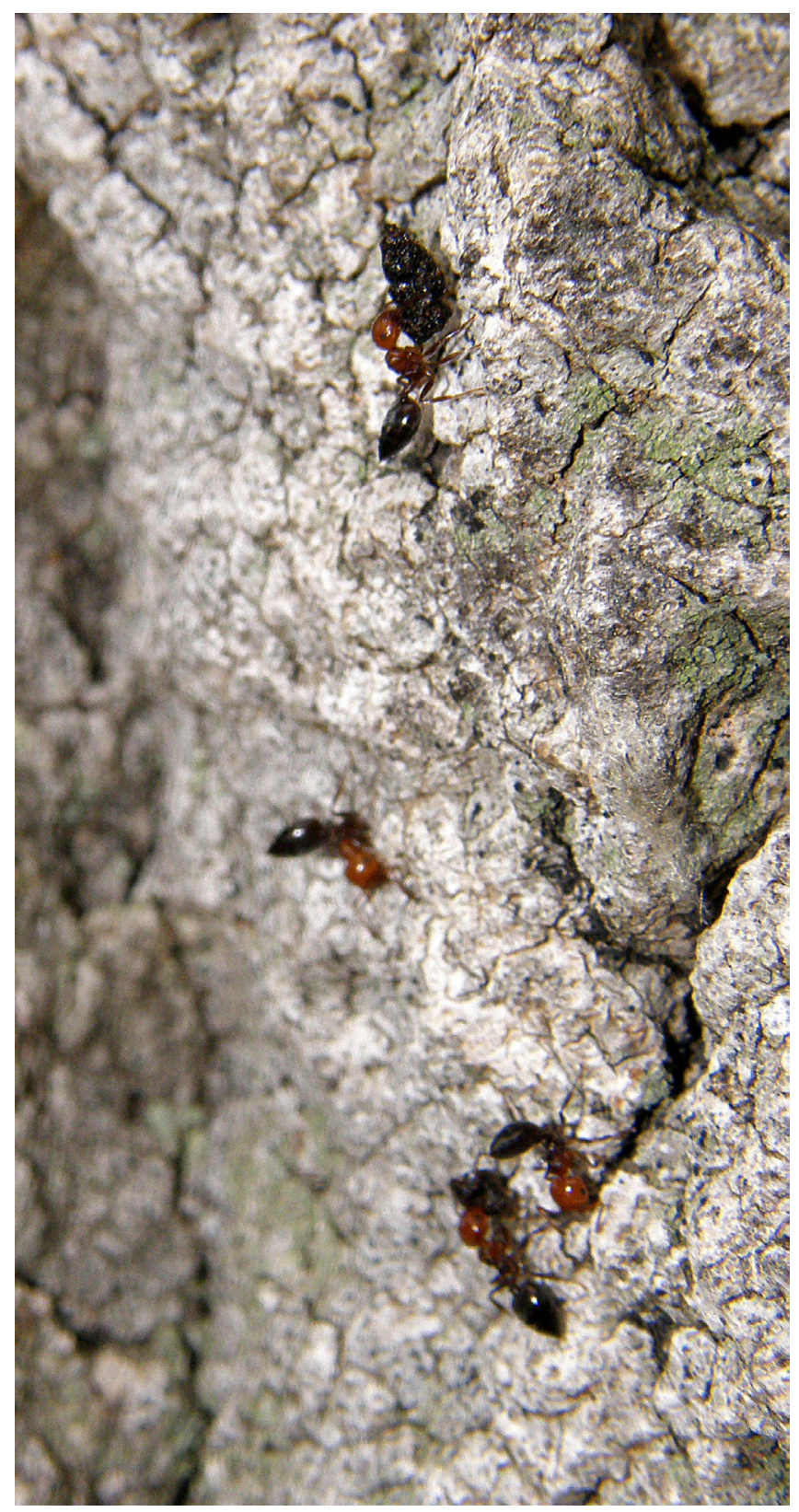

Fig. 1 - Hormiga roja transportando el guano por el árbol hasta su nido.

ejemplo: en España, Tinaut (2001) encontró una especie de hormiga (Hypoponera ragusai Emery, 1894), de distribución circunmediterránea, en tres cavidades andaluzas. Dicho autor observó las hormigas asociadas frecuentemente a depósitos de guano, aunque por el momento se desconoce si realmente se alimentan de éste o de otros organismos que viven en el mismo. Moulds (2006) observó hormigas cavernícolas australianas (Iridomyrmex purpureus Smith, 1858), recogiendo guano de murciélagos (Vespadelus findlaysoni Kitchener, Jones \& Caputi, 1987) en el interior de cavidades y transportándolo a su nido. No obstante, hasta ahora no se había observado este comportamiento fuera de cavidades.

La hormiga roja es una especie común, que se distribuye por Europa y Asia occidental (Social Insects Specialist Group 1996) y es un insecto oportunista, que puede construir sus nidos tanto en el suelo como en los árboles. Es omnívoro, aunque principalmente carnívoro, devorando áfidos, dípteros y larvas de himenópteros que caza o recoge muertos; en ciertas ocasiones puede alimentarse de las excreciones azucaradas de los áfidos y la savia de árboles (Skinner 1980).

Aunque hasta ahora no se le había observado aprovechando el guano de murciélagos, éste constituye un importante recurso alimenticio, utilizado por numerosos invertebrados que a su vez mantienen comunidades más diversas (Peck 1982, Ferreira \& Martins 1999, Webster \& Whitaker 2005, etc.) Se podría suponer por tanto que las hormigas rojas observadas recogían el guano de los murciélagos como alimento y lo transportaban a su nido situado en el álamo, al igual que también recogen los cadáveres de otros insectos muertos entre la vegetación. Teniendo en cuenta la elevada tasa metabólica de los murciélagos de Cabrera, y por tanto la gran cantidad de guano que produce una colonia, estos mamíferos podrían ofrecer un importante suministro de alimento a dichos invertebrados. La ausencia de otras observaciones similares en esta hormiga, cuyas poblaciones son abundantes y muy estudiadas, sugiere que este comportamiento pueda ser ocasional, fruto del oportunismo de estos insectos.

Por otro lado, los murciélagos de Cabrera se alimentan fundamentalmente de pequeños dípteros, mientras que los himenópteros constituyen únicamente entre el 2 y el $3 \%$ de sus presas (Barlow 1997, Bartonička et al. 2008). Ello permite deducir que en este caso, las hormigas rojas podrían resultar muy beneficiadas por los murciélagos de Cabrera, al disponer de un recurso alimenticio abundante y de fácil acceso, sin que ello entrañe un riesgo relevante.

\section{REFERENCIAS}

Alchlde J. T. 2000. Desaparece una importante colonia arborícola. Barbastella 1: 10-11.

BARLOw K. E. 1997. The diets of two phonic types of the bat Pipistrellus pipistrellus y Britain. J. Zool. Lond. 243: 597-609. DOI: https://dx.doi. org/10.1111/j.1469-7998.1997.tb02804.x

BARTONiČKA T., Řehák Z. \& ANDrEAS M. 2008. Diet composition and foraging activity of Pipistrellus pygmaeus in a floodplain forest. Biologia 63 (2): 1-7. DOI: https://dx.doi.org/10.2478/s11756-008-0034-y

Ferreira R. L., Martins R. P. 1999. Trophic structure and natural history of bat guano invertebrate communities, with special reference to Brazilian caves. Tropical Zoology 12: 231-252.

Moulds T. 2006. The first Australian record of subterranean guano-collecting ants. Helictite 39 (1): 3-4.

PECK S. B. 1982. A contribution to the knowledge of the invertebrate cave faunas of Venezuela: invertebrate faunas of tropical American caves, part 4. J. Speleol. 12: $75-81$.

SkINNER G. J. 1980. The feeding habits of the woodant, Formica rufa (Hymenoptera: Formicidae), in limestone woodland in North-West England. Journal of Animal Ecology 49: 417-433. 
Social InSeCts Specialist Group 1996. Formica rufa. In: IUCN 2013. IUCN Red List of Threatened Species. Version 2013.2. <www.iucnredlist.org>. Downloaded on 1 February 2014.
Tinaut T. 2001. Hypoponera ragusai (Emery, 1985) a cavernicolous ant new for the Iberian Peninsula (Hymenoptera, Formicidae). Graellsia 57 (1): 3-8.

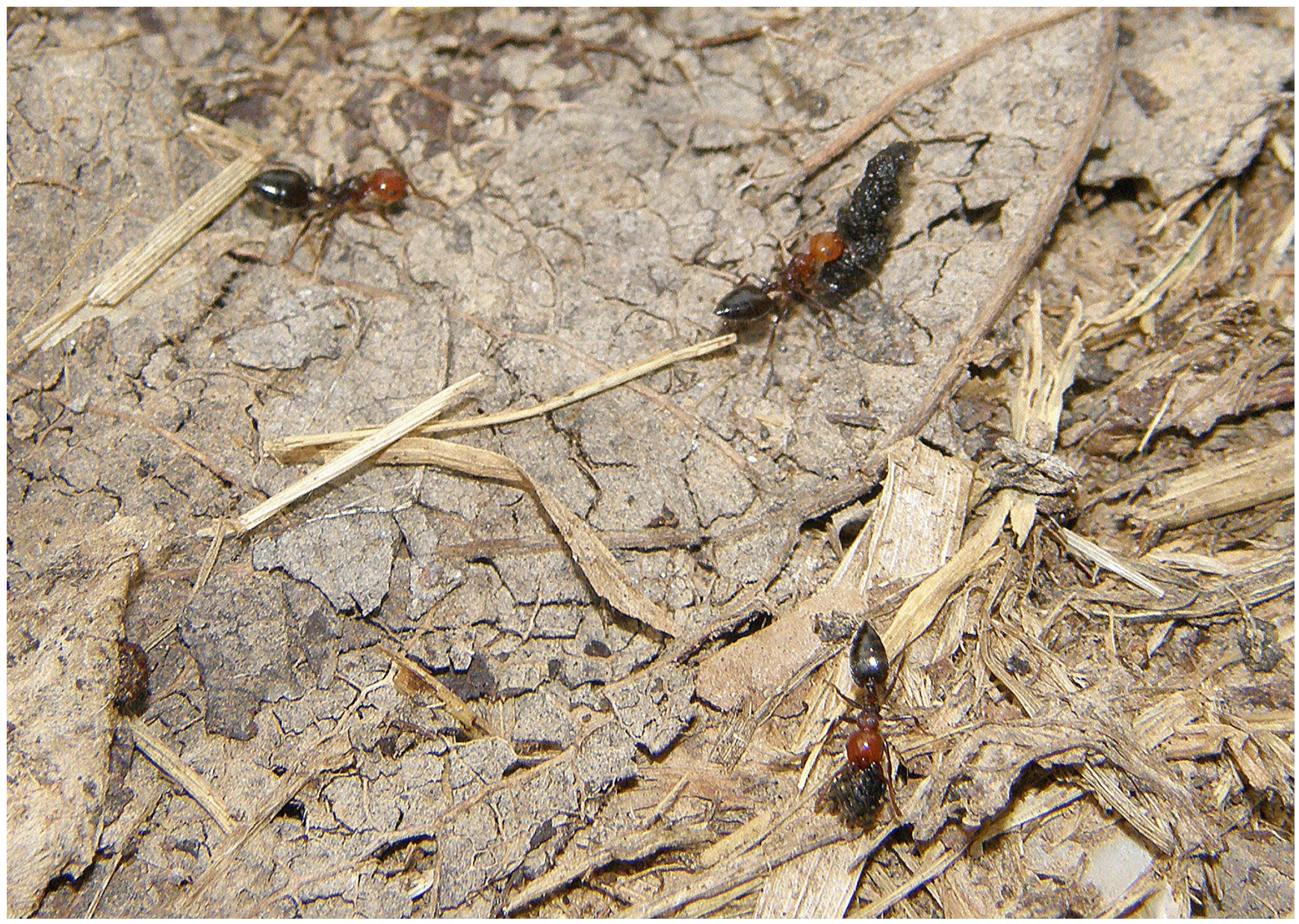

Fig. 2 - Hormiga roja (F. rufa) recogiendo guano de murciélagos de Cabrera (P. pygmaeus) del suelo. 